\title{
Síndrome de ovario poliquístico y embarazo
}

\author{
TERESA SIR-PETERMANN ${ }^{1}$, AMANDA LADRÓN DE GUEVARA ${ }^{1}$, \\ ANA CLAUDIA VILLARROEL ${ }^{2}$, JESSICA PREISLER ${ }^{1}$, \\ BÁRBARA ECHIBURÚ ${ }^{1, a}$, SERGIO RECABARREN ${ }^{3, b}$

\section{Polycystic ovary syndrome and pregnancy}

Polycystic ovary syndrome (PCOS) is a common endocrine metabolic dysfunction closely associated with insulin resistance and obesity, which predisposes to pregnancy complications and prenatal programming of the offspring. The aim of this review is to report our experience in PCOS patients who became pregnant and were followed during the whole pregnancy. Firstly, we analyzed the effect of pregnancy on PCOS pathophysiology and secondly the role of PCOS in pregnancy outcomes. Regarding the first point, during normal pregnancy a progressive insulin resistance, serum lipid changes and an increase in androgen levels is observed, which is exacerbated in the PCOS condition. This adverse intrauterine environment could have a prenatal programming effect with detrimental consequences for female or male fetuses. Regarding the second point, PCOS is associated with an increased risk for maternal complications such as gestational diabetes (GDM) and pregnancy-induced hypertension. Moreover, these adverse pregnancy outcomes are more frequently associated with an increase in low birth weight and high birth weight newborns. According to our clinical experience, PCOS patients who became pregnant and were not treated with metformin during the whole pregnancy, showed a higher prevalence of gestational diabetes and SGA newborns, which was improved with metformin treatment. In summary, pregnancy may constitute a period in which an abnormal condition is established or aggravated in the fetus of a PCOS mother. Moreover, PCOS enhanced adverse obstetric and neonatal outcomes.

(Rev Med Chile 2012; 140: 919-925).

Key words: Birth weight; Diabetes gestational; Polycystic ovary syndrome; Pregnancy complications.

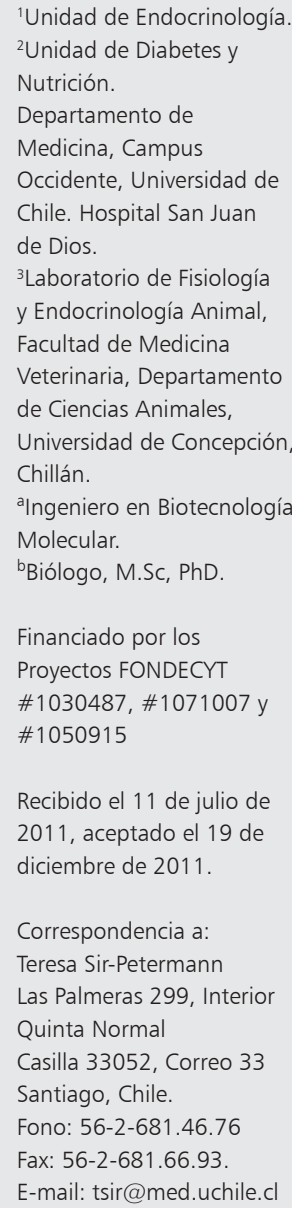

$\mathrm{E}$ 1 síndrome de ovario poliquístico (SOP), es una disfunción endocrino-metabólica heterogénea de alta prevalencia $(5-10 \%)^{1}$, caracterizada por oligo-ovulación e hiperandrogenismo, lo que compromete la función reproductiva de la mujer. Su etiología es incierta y se encuentra en estrecha asociación a la resistencia insulínica (RI), la que juega un papel preponderante en su fisiopatología y en las consecuencias metabólicas a largo plazo, entre las que destacan el desarrollo precoz de síndrome metabólico, diabetes tipo 2 y enfermedad cardiovascular ${ }^{2}$.
Las manifestaciones clínicas del SOP son heterogéneas y varían de acuerdo a la edad de la paciente. Se ha propuesto que este trastorno acompañaría a la mujer durante toda la vida, pudiendo manifestarse desde etapas muy tempranas del desarrollo sexual hasta la senectud ${ }^{3-6}$.

El SOP se caracteriza por presentar una morfología ovárica típica con múltiples folículos en crecimiento, aumento del estroma ovárico e hipersecreción de andrógenos, lo que sugiere que las alteraciones de la esteroidogénesis y de la foliculogénesis serían eventos centrales de este 
síndrome. Además, la hiperinsulinemia está involucrada en la fisiopatología del SOP tanto en sus aspectos metabólicos como reproductivos. La hiperinsulinemia estimula el desarrollo de folículos antrales y aumenta la sensibilidad de las células de la granulosa a FSH lo que conlleva a un aumento del número de folículos y del volumen ovárico ${ }^{7}$.

La disfunción de la esteroidogénesis ovárica se caracteriza por una excesiva producción de andrógenos por la teca de los folículos ováricos debido a una hiperactividad de la enzima citocromo p450c17 (CYP17) ${ }^{8}$. El aumento de la LH e insulina en el SOP llevan a una sobreexpresión enzimática, conduciendo a una mayor producción de andrógenos intraováricos los que en conjunto con factores paracrinos locales, alteran el desarrollo folicular. Estudios in vitro han establecido que la insulina actúa sinérgicamente con la LH en la síntesis de testosterona por las células tecales del ovario en mujeres normales y con SOP ${ }^{9}$. Clínicamente la disfunción de la esteroidogénesis puede reconocerse a través de un test de análogo de GnRH, lo cual permite evaluar la función gonadal y es la mejor manera de identificar al ovario como la fuente de una excesiva secreción de andrógenos ${ }^{10}$.

Respecto a la disfunción de la foliculogénesis, se ha podido establecer, mediante estudios ultrasonográficos y biopsias ováricas, que las pacientes con SOP presentan un pool de folículos en crecimiento 2-3 veces superior al de las mujeres sanas. La histología del SOP se caracteriza por un aumento de folículos preantrales y antrales pequeños y un mayor reclutamiento folicular ${ }^{11}$. Esta situación se acompaña además, de una detención del proceso de selección folicular, lo que explica la ausencia de ovulación ${ }^{12}$. Por lo tanto, en el SOP habría mayor reclutamiento y una menor selección, lo que mantiene un aumento del pool de folículos en crecimiento productores de andrógenos ${ }^{13}$. Esta masa folicular aumentada inhibe, mediante un efecto paracrino, la iniciación del crecimiento del resto de los folículos primordiales ${ }^{12}$. Lo anterior, asociado a una disminución del fenómeno de apoptosis por efecto androgénico, hace que la velocidad de depleción de folículos en estas pacientes disminuya, lo que aumenta la reserva ovárica por una fracción de folículos en crecimiento deteni$\operatorname{dos}^{14}$. Se ha establecido además, que las pacientes con SOP presentan concentraciones mayores de hormona anti-mülleriana $(\mathrm{AMH})$ e inhibina $\mathrm{B}$ comparados con mujeres no $\mathrm{SOP}^{15}$, las que cons- tituyen marcadores séricos de la reserva ovárica durante el período fértil ${ }^{16}$.

Dentro de la vida reproductiva de la mujer existen instancias en que el eje gonadal femenino presenta fases de quietud o frenación y de reactivación, estas ocurren en la prepubertad y en el embarazo-lactancia. El estudio del SOP durante estos períodos puede dar una orientación sobre su etiopatogenia.

\section{Cambios hormonales y metabólicos durante el embarazo normal}

En esta etapa, se producen modificaciones del metabolismo lipídico y de los carbohidratos que tienen como objetivo asegurar una entrega continua de nutrientes al feto, independiente de que la ingesta de alimentos por la madre sea intermitente. Estudios longitudinales durante la gestación, muestran una mayor respuesta insulínica a los nutrientes a pesar de un mínimo deterioro en la tolerancia a la glucosa, lo que es consistente con un aumento progresivo de la resistencia insulíni$\mathrm{Ca}^{17}$. Simultáneamente, se presenta un aumento progresivo del colesterol total, colesterol-LDL, colesterol-HDL y de los triglicéridos ${ }^{18}$.

\section{¿Cómo influye el embarazo sobre el SOP?}

Existe escasa información al respecto. Partiendo de la base que durante el embarazo se produce una frenación de la secreción gonadotrópica por las hormonas placentarias, la hipersecreción de LH debería quedar abolida y, por lo tanto, desde ese punto de vista el embarazo podría tener un efecto beneficioso sobre el SOP. Sin embargo, dado que el embarazo es un estado fisiológico de resistencia insulínica (RI), es probable que en aquellas pacientes con SOP y RI, el embarazo constituya un factor agravante de la insulinorresistencia pre-existente, pudiendo desencadenar una diabetes gestacional. Por lo tanto, el impacto del embarazo sobre cada caso particular dependerá del trastorno fisiopatológico predominante, este concepto nos motivó a desarrollar una línea de investigación que ha tenido una gran productividad y reconocimiento por la comunidad científica.

En los últimos años, se ha propuesto que factores ambientales, ya sea durante la vida prenata ${ }^{19}$ 
o postnatal ${ }^{20}$, llevarían a una expresión clínica y bioquímica del síndrome en la vida adulta. A este respecto, surge un nuevo concepto y de gran relevancia que vincula la etiopatogenia del SOP con los eventos que se producen durante la vida prenatal.

Entre los eventos deletéreos que se producen durante la vida intrauterina cabe mencionar al retardo del crecimiento intrauterino el que dará origen a niños pequeños para la edad gestacional (PEG) y a la exposición prenatal a andrógenos (EPA). Ambos han sido relacionados con la etiopatogenia del $\mathrm{SOP}^{19,21}$. Se ha planteado que los niños PEG tienen mayor riesgo de desarrollar síndrome metabólico en la vida adulta y se ha sugerido además, una relación entre el bajo peso de nacimiento y el desarrollo posterior de $\mathrm{SOP}^{22}$. A este respecto, hemos podido establecer que el antecedente de PEG es mayor en mujeres con SOP y que la prevalencia de PEG es significativamente mayor en los niños nacidos de madres $\mathrm{SOP}^{23,24}$. Respecto al segundo determinante prenatal, se ha planteado que la EPA estaría relacionada con la etiopatogenia del SOP. Estudios en modelos animales (monas y ovejas) establecen que las hembras androgenizadas por una exposición prenatal a andrógenos durante la vida intrauterina, como consecuencia de la administración exógena de testosterona a las madres, desarrollan rasgos postpuberales típicos de $\mathrm{SOP}^{25,26}$. En humanos, se ha observado que mujeres portadoras de una deficiencia de la 21-hidroxilasa se autoandrogenizan durante la vida fetal desarrollando un SOP secundario en la vida postnatal. Estas observaciones sugieren que la EPA de origen materno y/o fetal sería un posible mecanismo etiopatogénico para el desarrollo de SOP. En el modelo animal, es la madre la que traspasa el exceso de andrógenos al feto mientras que en el modelo humano (deficiencia de la 21-hidroxilasa) es la adrenal fetal la que produce un exceso de andrógenos generando una "autoandrogenización" y un SOP secundario. Estos resultados sugieren que la exposición prenatal a andrógenos podría explicar mucho de los rasgos típicos del SOP. En este sentido cobra gran interés las observaciones efectuadas por nuestro grupo en mujeres con SOP en amenorrea de la lactancia las cuales presentaban ovarios aumentados de volumen y una mayor concentración de androstenediona que las nodrizas normales ${ }^{27}$. Esto permitió inicialmente plantear que los ovarios de estas mujeres permanecieron esteroidogénicamente activos durante el embarazo manteniendo una secreción androgénica elevada la que eventualmente podría haber androgenizado al feto. Posteriormente, establecimos que las embarazadas con SOP efectivamente presentan niveles elevados de andrógenos pudiendo constituir una fuente de exceso de andrógenos para el feto, asemejando al modelo experimental ${ }^{21}$.

Además, de lo anteriormente expuesto, debemos recordar que el feto femenino o masculino de una madre SOP se va a desarrollar en un ambiente materno hiperinsulinémico ${ }^{21,28}$, lo cual también podría influir en la concentración de esteroides sexuales.

En síntesis podemos decir, que de acuerdo a nuestros estudios, la embarazada con SOP presenta elevadas concentraciones de andrógenos, mayores concentraciones de insulina y triglicéridos, menores concentraciones de adiponectina (proteína secretada por el adipocito con efecto sensibilizante a la insulina) y una exacerbación de la IR fisiológica del embarazo que podrían actuar como factores de reprogramación fetal ${ }^{21,28,29}$.

Basado en estas observaciones en los últimos diez años nuestro quehacer científico ha estado enfocado en estudiar la función reproductiva y metabólica de hijas e hijos de mujeres portadoras de un SOP clásico durante diferentes etapas del desarrollo sexual.

Cabe hacer notar que esta es una experiencia única en el mundo y reconocida como tal internacionalmente, por lo que no es factible hacer comparaciones con otros grupos.

Desde el punto de vista reproductivo, hemos demostrado que tanto las hijas como los hijos nacidos de madres con SOP presentan elevadas concentraciones de hormona anti-mülleriana $(\mathrm{AMH})$ durante el período prepuberal (infancia temprana y niñez), lo que sugiere una alteración del desarrollo folicular en las niñas y de la función de la célula de Sertoli en los niños ${ }^{3,30,31}$. Debido a que las células de la granulosa y de Sertoli tienen un origen embriológico común, se podría especular que factores genéticos y/o ambientales afectarían una línea celular específica durante el desarrollo de la gónada fetal. Otra observación interesante que hemos podido establecer es que las alteraciones metabólicas preceden al hiperandrogenismo en las hijas de madres con SOP y que los rasgos bioquímicos del SOP debutan en etapa Tanner IV del desarrollo puberal con aumento de la testosterona y una hiperrespuesta de $\mathrm{LH}$ al test 
de análogo de $\mathrm{GnRH}^{6}$. Desde el punto de vista metabólico, las hijas de madres con SOP muestran bajas concentraciones de adiponectina (proteína sensibilizante a la insulina que se origina en el adipocito); y un aumento de las concentraciones de insulina y triglicéridos ${ }^{5}$, mientras que los hijos presentan sobrepeso desde la infancia y desarrollan RI a medida que se hacen más adultos ${ }^{32}$. Estos datos, en conjunto, sugieren que los hijos e hijas de madres con SOP constituyen un grupo de alto riesgo para desarrollar alteraciones metabólicas y reproductivas a lo largo de la vida.

En conjunto, nuestros resultados muestran que existiría un probable fenómeno de reprogramación fetal de los hijos de madres SOP, que se evidencia tanto por una mayor frecuencia de niños PEG como por cambios en la función reproductiva y metabólica de estos niños. Debido a que en humanos es difícil separar el aspecto genético del ambiental los modelos experimentales de androgenización prenatal son de gran relevancia. En este sentido, estudios paralelos en un modelo ovino han contribuido a corroborar varias de las observaciones efectuadas en los hijos e hijas de estas pacientes, dando soporte experimental a la hipótesis de la reprogramación fetal por andrógenos. Las hijas nacidas de madres androgenizadas desarrollan rasgos que asemejan al SOP humano como anovulación, hiperandrogenismo, hipersecreción de estradiol en el test de análogo de $\mathrm{GnRH}$, alteración de la secreción de LH, insulino resistencia, retardo del crecimiento intrauterino y morfología poliquística de los ovarios. Por otro lado, los machos presentan alteraciones espermáticas, de la sensibilidad del eje hipófisis-testículo al test de análogo de GnRH y de la célula de Sertoli ${ }^{33-35}$.

\section{¿Cómo influye el SOP sobre el embarazo?}

Se han descrito una serie de complicaciones de la mujer embarazada con SOP, entre las cuales destacan: mayor tasa de abortos en el primer trimestre de embarazo, pre-eclampsia, diabetes gestacional, partos prematuros y mortinatos, macrosomía o niños nacidos grandes para la edad gestacional (GEG) y recién nacidos de bajo peso para la edad gestacional $(\mathrm{PEG})^{36}$.

A este respecto, realizamos un estudio clínico titulado "Síndrome de ovario poliquístico y embarazo: experiencia clínica"29. En dicho estudio se incluyeron 150 pacientes mujeres con SOP entre el año 2000 y 2005 que deseaban embarazo y que no tenían otras causas de infertilidad. Se logró el embarazo en 120 mujeres. Todas las pacientes al momento del diagnóstico de SOP e IR recibieron indicaciones de dieta de bajo índice glicémico, ejercicio físico consistente en media hora de caminata diaria y metformina en una dosis promedio de $1.500 \mathrm{mg} /$ día. La tasa de ovulación fue de $90 \%$ y la tasa de embarazo fue de $80 \%$. La prevalencia de diabetes gestacional fue significativamente mayor en mujeres con SOP en comparación con las controles (34,3 vs 5\%). El índice de masa corporal (IMC) también fue más elevado en mujeres con SOP. La hipertensión durante el embarazo fue levemente mayor en estas mujeres.

La concentración sérica de glucosa y de insulina basal y post carga en las semanas 10 a 16 y 22 a 28 de gestación fue mayor en mujeres con SOP en comparación a las embarazadas controles. Además, las mujeres con SOP mostraron una menor sensibilidad a la insulina durante el tercer trimestre de embarazo.

En cuanto a las características de los recién nacidos, las mujeres con SOP presentaron una mayor prevalencia, pero no significativa de niños GEG y PEG.

Este estudio muestra que el tratamiento enfocado en combatir la RI fue efectivo en recuperar la fertilidad en las mujeres con SOP. No obstante, los niveles de insulina post carga se mantuvieron significativamente más altos, durante toda la gestación, en las embarazadas con SOP en comparación a las embarazadas normales.

En síntesis: Si bien el tratamiento orientado al manejo de la RI en la paciente con SOP permite recuperar la fertilidad, lograr un embarazo y evitar los abortos en el primer trimestre, no se logra prevenir con medidas no farmacológicas la diabetes gestacional, la hiperinsulinemia, ni los recién nacidos PEG.

Debido a lo anterior se diseñó una segunda estrategia en la cual se utilizó metformina durante todo el embarazo con el fin de modificar las alteraciones endocrino-metabólicas que presentan estas pacientes durante el embarazo.

Actualmente, algunos estudios han establecido que el uso de metformina durante el embarazo es seguro para el feto y se plantea mantenerlo en las pacientes con SOP durante todo el embarazo para disminuir la tasa de abortos espontáneos, prevenir 
el desarrollo de diabetes gestacional, el síndrome hipertensivo del embarazo y la androgenización prenatal del hijo de madre con $\mathrm{SOP}^{37,38}$. Hasta el momento, no se ha descrito un efecto deletéreo de la metformina sobre el peso corporal, talla y cambios en el desarrollo psicomotor de los recién nacidos $^{39}$

De acuerdo a nuestra experiencia, las mujeres que recibieron metformina durante el embarazo no presentaron niños PEG ni GEG, la prevalencia de diabetes gestacional disminuyó significativamente de $36 \%$ a $14 \%$, y las hijas presentaron una menor concentración de AMH que las hijas de madres no tratadas, alcanzando valores de $\mathrm{AMH}$ sérica comparables a los observados en hijas de mujeres controles. En las madres, se observó una disminución significativa de los niveles de insulina y andrógenos séricos y un aumento de la sensibilidad insulínica ${ }^{40}$.

Por lo tanto, la administración de metformina durante el embarazo a la madre con SOP, parece tener un efecto favorable sobre las alteraciones endocrino-metabólicas que presentan estas pacientes, disminuyendo el desarrollo de diabetes gestacional y previniendo el exceso de andrógenos para el feto, lo que se traduce en una disminución de niños con extremos de peso al nacer y una disminución de la masa folicular de las hijas.

Las dos cohortes estudiadas, de mujeres tratadas y no tratadas tenían un SOP clásico con insulinorresistencia que es la forma más severa de esta entidad. Según algunos autores el aumento del riesgo para eventos obstétricos y neonatales adversos varía ampliamente de acuerdo con los diferentes fenotipos y rasgos de este síndrome.

La metformina, probablemente estaría indicada en este tipo de pacientes por el mayor riesgo de diabetes gestacional, síndrome hipertensivo del embarazo y los extremos de peso al nacer de los recién nacidos. Es probable que otras formas menos severas del síndrome puedan ser manejadas con medidas no farmacológicas como dieta y ejercicio durante el embarazo.

En síntesis, de acuerdo a nuestra experiencia, podemos decir que el embarazo no tiene un efecto beneficioso sobre el SOP materno y por el contrario, puede ofrecer una instancia en que se inicie o agrave una condición preexistente en la hija o hijo de una madre con SOP. Además, el SOP aumenta el riesgo de patologías del embarazo, perinatales y obstétricas.
Por lo tanto, una forma de evitar que se perpetúe este síndrome es el manejo adecuado de la madre con SOP durante el embarazo.

Agradecimientos: Deseamos agradecer a los miembros académicos y no académicos del Laboratorio de Endocrinología y Metabolismo de la Facultad de Medicina Occidente de la Universidad de Chile por su colaboración y al Dr. García de los Ríos por su revisión crítica del manuscrito.

\section{Referencias}

1. Knochenhauer ES, Key TJ, Kashsar-Miller M, Waggoner W, Boots LR, Azziz R. Prevalence of polycystic ovary syndrome in unselected black and white women of the southeastern United States: a prospective study. J Clin Endocrinol Metab 1998; 71: 3078-82.

2. Carmina E, Lobo RA. Polycystic ovary syndrome (PCOS): arguably the most common endocrinopathy is associated with significant morbidity in women. J Clin Endocrinol Metab 1999; 84: 1897-9.

3. Crisosto N, Codner E, Maliqueo M, Echiburú B, Sánchez F, Cassorla F, et al. Anti-Müllerian hormone levels in peripubertal daughters of women with polycystic ovary syndrome. J Clin Endocrinol Metab 2007; 92: 2739-43.

4. Pasquali R, Gambineri A. Polycystic ovary syndrome: a multifaceted disease from adolescence to adult age. Ann N Y Acad Sci 2006; 1092: 158-74.

5. Sir-Petermann T, Maliqueo M, Codner E, Echiburú B, Crisosto N, Pérez V, et al. Early metabolic derangements in daughters of women with polycystic ovary syndrome. J Clin Endocrinol Metab 2007; 92: 4637-42.

6. Sir-Petermann T, Codner E, Pérez V, Echiburú B, Maliqueo M, Ladrón de Guevara AH, et al. Metabolic and reproductive features before and during puberty in daughters of women with polycystic ovary syndrome. J Clin Endocrinol Metab 2009; 94: 1923-30.

7. Fulghesu AM, Villa P, Pavone V, Guido M, Apa R, Caruso A, et al. The impact of insulin secretion on the ovarian response to exogenous gonadotropins in polycystic ovary syndrome. J Clin Endocrinol Metab 1997; 82: 644-8.

8. Gilling-Smith C, Willis DS, Beard RW, Franks S. Hypersecretion of androstenedione by isolated theca cells from polycystic ovaries. J Clin Endocrinol Metab 1994; 79: 1158-65.

9. Cara JF, Rosenfield RL. Insulin-like growth factor I and insulin potentiate luteinizing hormone-induced 
androgen synthesis by rat ovarian thecal-interstitial cells. Endocrinology 1988; 123: 733-9.

10. Piltonen T, Koivunen R, Ruokonen A, Tapanainen JS. Ovarian age-related responsiveness to human chorionic gonadotropin. J Clin Endocrinol Metab 2003; 88: 3327 32.

11. Hughesdon PE. Morphology and morphogenesis of the Stein-Leventhal ovary and of so-called "hyperthecosis". Obstet Gynecol Surv 1982; 37: 59-77.

12. Webber LJ, Stubbs S, Stark J, Trew GH, Margara R, Hardy K, et al. Formation and early development of follicles in the polycystic ovary. Lancet 2003; 362: 1017-21.

13. Jonard S, Dewailly D. The follicular excess in polycystic ovaries, due to intraovarian hyperandrogenism, may be the main culprit for the follicular arrest. Hum Reprod 2004; 10: 107-17.

14. Takayama K, Fukaya T, Sasano H, Funayama Y, Susuki $\mathrm{T}$, Takaya R, et al. Immunohistochemical study of steroidogenesis and cell proliferation in polycystic ovarian syndrome. Hum Reprod 1996; 11: 1387-92.

15. Piltonen T, Morin-Papunen L, Koivunen R, Perheentupa A, Ruokonen A, Tapanainen J. Serum anti-mullerian hormone levels remain high until late reproductive age and decrease during metformin therapy in women with polycystic ovary syndrome. Hum Reprod 2005; 20: 1820-6.

16. Sowers MR, Eyvazzadeh AD, McConnell D, Yosef M, Jannausch ML, Zhang D, et al. Anti-Mullerian Hormone and Inhibin B in the Definition of Ovarian Aging and the Menopause Transition. J Clin Endocrinol Metab 2008; 93: 3478-83.

17. Catalano PM, Tyzbir ED, Roman NM, Amini SB, Sims EA. Longitudinal changes in insulin release and insulin resistance in nonobese pregnant women. Am J Obstet Gynecol 1991; 165: 1667-72.

18. Butte NF. Carbohydrate and lipid metabolism in pregnancy: normal compared with gestational diabetes mellitus. Am J Clin Nutr 2000; 71: 1256S-61S.

19. Xita N, Tsatsoulis A. Fetal Programming of Polycystic Ovary Syndrome by Androgen Excess: Evidence from Experimental, Clinical and Genetic Association Studies. J Clin Endocrinol Metab 2006; 91: 1660-6.

20. Gambineri A, Pelusi C, Vicennati V, Pagotto U, Pasquali R. Obesity and the polycystic ovary syndrome. Int J Obes Relat Metab Disord 2002; 26: 883-96.

21. Sir-Petermann T, Maliqueo M, Angel B, Lara HE, PérezBravo F, Recabarren SE. Maternal serum androgens in pregnant women with polycystic ovarian syndrome: possible implications in prenatal androgenization. Hum Reprod 2002; 17: 2573-9.

22. Sir-Petermann T, Hitschfeld C, Codner E, Maliqueo
M, Ávila A, Echiburú B, et al. Gonadal function in low birth weight infants. J Pediatr Endocrinol Metab 2007; 20: 405-14.

23. Benítez R, Sir-Petermann T, Palomino A, Angel B, Maliqueo M, Pérez F, et al. Prevalencia familiar de patologías metabólicas en pacientes con síndrome de ovario poliquístico. Rev Med Chile 2001; 129: 707-12.

24. Sir-Petermann T, Hitchsfeld C, Maliqueo M, Codner E, Echiburú B, Gacitúa R, et al. Birth weight in offspring of mothers with polycystic ovary syndrome. Hum Reprod 2005; 20: 2122-6.

25. Padmanabhan V, Manikkam M, Recabarren S, Foster D. Prenatal testosterone excess programs reproductive and metabolic dysfunction in the female. Mol Cell Endocrinol 2006; 246: 165-74.

26. Abbott DH, Tarantal AF, Dumesic DA. Fetal, infant, adolescent and adult phenotypes of polycystic ovary syndrome in prenatally androgenized female rhesus monkeys. Am J Primatol 2009; 71: 776-84.

27. Sir-Petermann T, Devoto L, Maliqueo M, Peirano P, Recabarren SE, Wildt L. Resumption of ovarian function during lactational amenorrhoea in breastfeeding women with polycystic ovary syndrome: endocrine aspects. Hum Reprod 2001; 16: 1603-10.

28. Sir-Petermann T, Echiburú B, Maliqueo M, Crisosto N, Sánchez F, Hitschfeld C, et al. Serum adiponectin and lipid concentrations in pregnant women with polycystic ovary syndrome. Hum Reprod 2007; 22: 1830-6.

29. Villarroel AC, Echiburú B, Riesco V, Maliqueo M, Cárcamo M, Hitschfeld C, et al. Síndrome de ovario poliquístico (SOP) y embarazo: Experiencia clínica. Rev Med Chile 2007; 135: 1530-1538.

30. Recabarren SE, Sir-Petermann T, Rios R, Maliqueo M, Echiburú B, Smith R, et al. Pituitary and testicular function in sons of women with polycystic ovary syndrome from infancy to adulthood. J Clin Endocrinol Metab 2008; 93: 3318-24.

31. Sir-Petermann T, Codner E, Maliqueo M, Echiburú B, Hitschfeld C, Crisosto N, et al. Increased anti-Müllerian hormone serum concentrations in prepubertal daughters of women with polycystic ovary syndrome. J Clin Endocrinol Metab 2006; 91: 3105-9.

32. Recabarren SE, Smith R, Ríos R, Maliqueo M, Echiburú $\mathrm{B}$, Codner E, et al. Metabolic profile in sons of women with polycystic ovary syndrome. J Clin Endocrinol Metab 2008; 93: 1820-6.

33. Recabarren SE, Padmanabhan V, Codner E, Lobos A, Durán C, Vidal M, et al. Postnatal developmental consequences of altered insulin sensitivity in female sheep treated prenatally with testosterone. Am J Physiol Endocrinol Metab 2005; 289: E801-6. 
34. Recabarren SE, Lobos A, Figueroa Y, Padmanabhan V, Foster DL, Sir-Petermann T. Prenatal testosterone treatment alters $\mathrm{LH}$ and testosterone responsiveness to GnRH agonist in male sheep. Biol Res 2007; 40: 32938.

35. Recabarren SE, Rojas-García PP, Recabarren MP, Alfaro VH, Smith R, Padmanabhan V, et al. Prenatal testosterone excess reduces sperm count and motility. Endocrinology 2008; 149: 6444-8.

36. Altieri P, Gambineri A, Prontera O, Cionci G, Franchina M, Pasquali R. Maternal polycystic ovary syndrome may be associated with adverse pregnancy outcomes. Eur J Obstet Gynecol Reprod Biol 2010; 149: 31-6.

37. Goldenberg N, Glueck C. Medical therapy in women with polycystic ovarian syndrome before and during pregnancy and lactation. Minerva Ginecol 2008; 60: 6375 .
38. De Leo V, Musacchio MC, Piomboni P, Di Sabatino A, Morgante G. The administration of metformin during pregnancy reduces polycystic ovary syndrome related gestational complications. Eur J Obstet Gynecol Reprod Biol 2011; 157: 63-6.

39. Glueck CJ, Goldenberg N, Pranikoff J, Loftspring M, Sieve L, Wang P. Height, weight, and motor-social development during the first 18 months of life in 126 infants born to 109 mothers with polycystic ovary syndrome who conceived on and continued metformin through pregnancy. Hum Reprod 2004; 19: 1323-30.

40. Crisosto N, Echiburú B, Maliqueo M, Pérez V, de Guevara AL, Preisler J, et al. Improvement of hyperandrogenism and hyperinsulinemia during pregnancy in women with polycystic ovary syndrome: possible effect in the ovarian follicular mass of their daughters. Fertil Steril 2012; 97: 218-24. 